\title{
Quadricuspid aortic valve repair
}

\section{Stefano Mastrobuoni, Gaby Aphram, Saadallah Tamer, Emiliano Navarra, Laurent De Kerchove, Gebrine El Khoury}

Department of Cardiovascular and Thoracic Surgery, St. Luc's Hospital, Catholic University of Louvain, Brussels, Belgium

Correspondence to: Stefano Mastrobuoni, MD, MPH. Cardiovascular and Thoracic Surgery, St. Luc's Hospital, Avenue Hippocrate 10, 1200 Brussels, Belgium. Email: stefano.mastrobuoni@uclouvain.be.

Submitted Mar 12, 2019. Accepted for publication May 06, 2019.

doi: 10.21037/acs.2019.05.05

View this article at: http://dx.doi.org/10.21037/acs.2019.05.05

\section{Clinical vignette}

A 47-year-old man was referred to our department for severe and symptomatic aortic regurgitation. The patient complained of shortness of breath (NYHA II-III) over the previous couple of months. He also presented with arterial hypertension, dyslipidaemia and a history of smoking. Physical examination revealed a diastolic murmur in the aortic and mitral valve areas. A transoesophageal ultrasound scan showed normal left ventricular function and severe aortic regurgitation on a quadricuspid valve. Further, the left ventricle was slightly dilated with an end-diastolic diameter (LVEDD of $61 \mathrm{~mm}$ ) but not aortic dilatation. A coronary angiogram did not show significant coronary lesions or anomalies. The patient was scheduled for elective repair of the aortic valve.

\section{Surgical technique}

\section{Preparation}

Standard approach was applied with the patient under general anesthesia in the supine position, mechanical ventilation, central venous line, invasive arterial pressure monitoring and trans-esophageal echo.

\section{Exposition}

Our standard approach is through median sternotomy. After incision of the pericardium, conventional cannulation for cardiopulmonary bypass (CPB) through the right atrium and distal ascending aorta is performed, unless the patient requires a concomitant arch replacement, in which case, we prefer cannulation of the right axillary artery in order to ensure antegrade cerebral perfusion during the open distal aorta replacement.

\section{Operation}

In standard elective procedures, after aortic cross clamp warm blood cardioplegia is delivered in the aortic root. If there is significant aortic regurgitation, the aorta is opened transversely $1 \mathrm{~cm}$ above the sino-tubular junction (STJ) and the cardioplegia given directly through two ostial cannulas. Cardioplegia is then repeated every 15 minutes. The aortic valve and root are then assessed to confirm the indication for valve repair or valve-sparing aortic root replacement in patients with dilated root (diameter $>45 \mathrm{~mm}$ ).

At this point, it is of paramount importance to carefully and systematically asses the valve leaflets with particular attention to the quality of tissue, cusp mobility, presence of fibrosis, calcifications or fenestration. The probability of valve repair with long-term durability is estimated at this point. Small fenestrations, as well as little calcifications or fibrosis, can be addressed during the repair and do not necessarily represent a contraindication to the valve repair or sparing procedures. However, severe calcifications or extensive fibrosis may compromise the durability of the repair. Large fenestrations of the cusp that require large patch may compromise the durability of the repair and may also contraindicate the procedure. Moreover, it is important to evaluate the height of the free margin of each cusp in order to identify cusp prolapse as the mechanism of regurgitation. The measurement of geometric and effective heights can be helpful for this purpose.

The surgical steps towards aortic valve repair with the "tricuspidization" technique are described below and 
presented in the video. First, three commissural traction stitches (4-0 polypropylene) are placed at the tip of each commissure. In the most common configuration of quadricuspid aortic valve (QAV) there is an accessory cusp next to a cusp (left coronary cusp in our case) that is smaller than the other two. A 6-0 polypropylene is passed through the middle of free edge of the accessory cusp and the smaller cusp. The rudimentary commissure is then detached from the aortic wall with small cut of Metzenbaum scissors. Detachment of the rudimentary commissure reveals that often the 2 cusps are partially fused below. Also, this maneuver increases cusp mobility. The raphe is eventually shaved, if particularly fibrotic. The left and accessory cusps are then sutured together with a 6-0 polypropylene following the vertical line of contact. A small pericardial patch may be needed to fill the gap after resection of the raphe. If the height of the free margin of the neo-cusp is lower than the 2 other cusps, a central plication can be applied to correct the prolapse.

The repair is usually stabilized with an annuloplasty. In this patient, we employed the sub-commissural annuloplasty (Cabroll stitches). However, in cases of external annuloplasty, the aortic root is prepared by separation from the surrounding structure down to the level of ventriculo-aortic junction (VAJ) leaving the coronary arteries attached to the aortic root (see video). Below each coronary artery, a tunnel is created with right-angle forceps in order to pass the band below the coronary vessels. Once the dissection is completed, 9-10 pledged stitches are placed below the valve along the basal ring. Below the left coronary artery, however, care must be taken to avoid injury to the circumflex coronary artery which can follow a vertical path close to the VAJ area. The 3 or 4 stitches of the VAJ anteriorly between the coronary arteries are passed first through the middle portion of the band. Afterwards, the band is pulled down and its two extremities are passed below each coronary artery. Finally, the remaining stitches are passed symmetrically on both ends of the band. The last stitch at the level of the non-coronary cusp is passed through each extremity of the band in order to make a true complete ring annuloplasty. In order to correctly reduce the size of the VAJ, the annuloplasty stitches are tied with a Hegar dilator inserted through the VAJ. The size of the dilator is 23 or 25 , depending on patient's body size.

After valve repair, it is critical to re-examine the leaflets for any residual prolapse, symmetry and the height and depth of coaptation before closing the aorta. Residual cusp prolapse can be usually treated with central plication or triangular resection. Finally, the aortotomy is closed with a 4-0 polypropylene. If the ascending aorta is dilated (diameter $>45 \mathrm{~mm}$ ), it is then replaced with a Dacron graft. If the ascending aorta is only mildly dilated (diameter between 40 and $45 \mathrm{~mm}$ ), the STJ can be downsized and remodeled with the suture of the aortotomy reinforced with an external felt band.

\section{Completion}

After weaning from CPB, the valve is thoroughly reassessed with the transesophageal echo.

\section{Comments}

QAV is an infrequent congenital anomaly that usually manifests with valvular disease in young patients. Hurwitz and Roberts were the first to describe the configurations of QAV (1). In the most common configurations, there is either a small cusp with a $4^{\text {th }}$ rudimentary accessory cusp (as in the case presented), or 3 cusps including 1 large conjoined cusp with a raphe (similar to the type 1 bicuspid aortic valve). Further, aortic insufficiency is the predominant form of disease in these cases (2). The mechanism of aortic regurgitation is either cusp restriction, as a result of incomplete fusion of the accessory cusp, or cusp prolapse of the conjoined cusp. Once the mechanism of regurgitation is clearly identified, whether cusp restriction (type 3 according to our classification) (3), or cusp prolapse (type 2), the repair can be successfully achieved applying the proper techniques. On one hand, cusp restriction can be corrected by detachment of the pseudo-commissure, resection of the fibrotic raphe and reapproximating the cusp edges, resulting in "tricuspidization" of the QAV (4) as in this case.

On the other hand, prolapse of the conjoined cusp or lack of coaptation in cases of QAV with 4 cusps of similar size can be corrected by central plication of the prolapsing cusp and closure of the commissures resulting in "bicuspidization" of the QAV (5).

Stabilization of the aortic annulus, particularly the VAJ, is an important adjunctive technique to ensure longterm durability. In cases of aortic root replacement, valve sparing with the reimplantation technique will provide the VAJ annuloplasty. In cases where the aortic root is not replaced, the annuloplasty can be carried out with the subcommissural stitches (Cabrol technique), external or internal ring. In case of external annuloplasty, as shown at the end of the video, an external flexible prosthetic band (a 
strip of Dacron graft or the Simplicity ${ }^{\circledR}$ band, Medtronic Inc.) is fixed around the VAJ.

Although repair of QAV has been reported in small series with medium-term follow-up, valve repair appears particularly appealing in these young patients in contrast to prosthetic valve replacement that carries the wellknown risk of prostheses-related complications in the longterm. Idrees and colleagues (2) from the Cleveland Clinic reported the largest series with 31 patients operated for QAV. Valve replacement was performed in 24 patients and repair in the remaining 7 . The authors observed that despite 21 patients having severe aortic regurgitation, most of them had severely degenerated cusps and the valve could be repaired only in 7 cases. The tricuspidization technique was the most used technique.

Since 2002, we have treated 10 patients with QAV. The mean age was $45 \pm 18$ years. Seven patients presented with severe aortic regurgitation, 2 with aortic aneurysm and regurgitation and 1 case valve stenosis. The patient with aortic stenosis underwent valve replacement with the pulmonary autograft, 7 patients had isolated valve repair, 1 valve-sparing root replacement with the reimplantation technique and 1 valve repair and ascending aorta replacement. We employed the tricuspidization technique in 8 cases and we modified the QAV making bicuspid in 1 case only. In 2 cases, a pericardial patch was used to fill the gap after resection of the raphe. Further, we performed an annuloplasty in 8 cases (5 with sub-commissural annuloplasty, 2 external band, 1 internal ring and 1 with the proximal suture line of the valve reimplantation). No patients died during surgery and at a mean follow-up of 5 years none have had reoperation on the aortic valve.

\section{Conclusions}

In conclusion, in the most common configuration of QAV with the presence of an accessory cusp and a rudimentary

Cite this article as: Mastrobuoni S, Aphram G, Tamer S, Navarra E, De Kerchove L, El Khoury G. Quadricuspid aortic valve repair. Ann Cardiothorac Surg 2019;8(3):433-435. doi: 10.21037/acs.2019.05.05 commissure causing aortic regurgitation, valve repair can be carried out with the tricuspidization technique without increased perioperative risk and is associated with excellent long-term results in terms of recurrence of regurgitation and reoperation on the valve. Nonetheless, due to this rare and challenging anatomy, a solid experience with valve repair as well as careful patient selection are necessary to successfully perform the procedure in this young cohort of patients.

\section{Acknowledgments}

None.

\section{Footnote}

Conflicts of Interest: The authors have no conflicts of interest to declare.

\section{References}

1. Hurwitz LE, Roberts WC. Quadricuspid semilunar valve. Am J Cardiol 1973;31:623-6.

2. Idrees JJ, Roselli EE, Arafat A, et al. Outcomes after repair or replacement of dysfunctional quadricuspid aortic valve. J Thorac Cardiovasc Surg 2015;150:79-82 .

3. Boodhwani M, de Kerchove L, Glineur D, et al. Repairoriented classification of aortic insufficiency: impact on surgical techniques and clinical outcomes. J Thorac Cardiovasc Surg 2009;137:286-94.

4. Schmidt KI, Jeserich M, Aicher D, et al. Tricuspidization of the quadricuspid aortic valve. Ann Thorac Surg 2008;85:1087-9.

5. Luciani GB, Morjan M, Faggian G, et al. Repair of quadricuspid aortic valve by bicuspidization: a novel technique. Interact Cardiovasc Thorac Surg 2010;11:348-50. 